\title{
Los inicios de la CONAMED
}

The beginnings of CONAMED

Héctor Fernández Varela Mejía*

Quisiera empezar por manifestar mi absoluta certeza y convicción en que la creación de la Comisión Nacional de Arbitraje Médico (CONAMED) fue uno de los grandes aciertos del gobierno del presidente Ernesto Zedillo, que ha permitido contribuir a fortalecer el derecho a la protección de la salud, a la mejoría constante de la calidad de la atención médica, a contener la magnitud de la medicina defensiva y los altos costos de los servicios profesionales por la necesidad de contratar onerosos seguros de práctica médica ante posibles demandas económicas por malpraxis.

En la segunda mitad del siglo XX fue cada vez más evidente el aumento en el número de casos de insatisfacción con los servicios médicos, tanto individuales como institucionales, y se apreció durante las campañas políticas para elección presidencial una creciente demanda para que dichas quejas fueran atendidas.

Ese fue el caso que se percibió en el proceso electoral de Miguel de la Madrid, por lo que el Dr. Guillermo Soberón, en su carácter de secretario de salud, propuso la creación de un Ombudsman de la salud. Sin embargo, dicha iniciativa fue mal interpretada por el gremio médico que la consideró una figura para sancionar a los profesionales de la medicina, por lo que se decidió cancelar su implementación. No obstante, fue evidente que las quejas de la población continuaron aumentando en los sexenios siguientes y al tomar posesión el presidente Zedillo instruyó a la Secretaría de Salud en diseñar un método para atender las inconformidades de los pacientes. Para ello se constituyó un grupo multidisciplinario que después de meses de análisis propuso crear una Procuraduría de la Salud, que nuevamente fue rechazada tanto por las instituciones de salud como por las organizaciones médicas tanto científicas como gremiales. Oposición que se mantuvo a pesar de la campaña para aclarar su carácter imparcial, que se desplegó en todo el territorio nacional. Fue entonces que el Dr. Juan Ramón de la Fuente, secretario de salud, propuso la creación de un organismo conciliador, capaz de ofrecer un estudio imparcial de cada caso, buscando el acuerdo entre las partes y de no lograrse éste, podría resolverse mediante un juicio arbitral al que se sometieran ambas partes.

Así fue como el secretario convocó nuevamente al grupo de trabajo, al cabo de numerosas reuniones en la que intervenimos médicos, abogados, expertos en derechos humanos, intelectuales y expertos en juicios arbitrales, tanto en materia laboral como comercial, integramos un proyecto que garantizaba la imparcialidad buscada. A esto siguió una etapa muy compleja en la que fue necesario dialogar con todos los organismos y dependencias que podrían objetar una invasión en sus atribuciones y facultades. Se discutió tanto con la Procuraduría General de la República como con la Suprema Corte de Justicia de la Nación, donde tuve oportunidad

* Primer Comisionado Nacional de Arbitraje Médico. Director General de Atención a la Salud, Universidad Nacional Autónoma de México.

\section{Correspondencia:}

HFVM, hfernandezvar@ unam.mx

Conflicto de intereses: No hay conflicto de intereses. Citar como: Fernández VMH. Los inicios de la CONAMED. Rev CONAMED. 2021; 26(4): 163-165. https://dx.doi. org/10.35366/102501 Financiamiento: No hay financiamiento.

Recibido: 15/10/2021. Aceptado: 15/10/2021. 
de tratar personalmente con los titulares de ambas instituciones. Se informó también a las Cámaras de Diputados y Senadores para contar con su apoyo, con la Comisión Nacional de Derechos Humanos (CNDH) para vencer su resistencia a la propuesta y con el resto de las instituciones involucradas hasta lograr un documento aceptado por todos y que constituyó la base del decreto presidencial mediante el cual se creó la CONAMED, con sus funciones y atribuciones, su estructura y estatus como órgano desconcentrado de la Secretaría de Salud.

No entro en materia de su contenido, ya que fue ampliamente difundido y publicado en el Diario Oficial de la Federación el lunes 03 de junio de 1996.

Fui propuesto al cargo de comisionado nacional de arbitraje médico por el Dr. Juan Ramón de la Fuente y designado el 05 de junio por el presidente de la República. En esa misma fecha fue integrado el Consejo de la Comisión Nacional de Arbitraje Médico constituido por figuras relevantes tanto jurídicas como médicas, intelectuales y reconocidos defensores de los derechos humanos. El consejo llevó a cabo su primera sesión ordinaria ese mismo día. Durante dicha sesión tomé posesión del cargo y se aprobaron también los nombramientos de los dos subcomisionados: Lic. Rafael Domínguez Morfín y la Dra. María Farell Campa.

Asimismo, el consejo aprobó el inicio de actividades para el día siguiente, jueves 06 de junio. No considero exagerar al afirmar que al carecer de antecedentes de un órgano de esta naturaleza, realizamos una verdadera obra de creación en muy diversos temas, tales como los procedimientos para la recepción de las quejas, flujogramas para la ruta a seguir en los diversos asuntos, la distribución de los espacios de trabajo para las direcciones generales, horarios de atención y de actividades, la forma de integrar los expedientes, los métodos de estudio de las quejas, criterios para su aceptación o rechazo al proceso de conciliación, o bien como referirlas a la instancia correspondiente según el carácter de la inconformidad, ya fuera a la CNDH, las oficinas para la atención de quejas de las instituciones de salud así como aquellos casos que correspondían directamente al Poder Judicial o a la autoridad sanitaria (Secretaría de Salud).

Es evidente que todo este conjunto de procedimientos y normas tendría que ser en parte imperfecto, inadecuado, inconveniente o incluso erróneo en algunos casos, dada la premura y la falta de antecedentes que afrontamos en su elaboración y por tanto, susceptible de modificaciones, perfeccionamiento o eliminación, como sin duda ha sucedido.

Para la estructuración de todas estas decisiones y muchas otras nos apoyamos en las tareas ya realizadas desde meses antes de la creación de la CONAMED, como fue la designación de los primeros colaboradores, la visita a inmuebles, la decisión del inmueble más adecuado y factible para la firma del contrato de arrendamiento por la Secretaría de Hacienda. Se realizaron las divisiones arquitectónicas del edificio, la adquisición de mobiliario, de equipos de cómputo, de insumos, vehículos y muchas otras tareas.

Pensamos que pasarían algunos días antes de que acudieran personas con inconformidades sobre la atención médica, pero sorpresivamente el mismo jueves 06 de junio se presentó un número importante de pacientes o familiares que desde luego atendimos y su número creció a lo largo de los meses; en aquel primer año el número de quejas admitidas fueron 2,071.

Facilitamos la presentación de las quejas al poder hacerse no sólo en forma personal, sino por correo o por vía telefónica.

Desde el inicio fue prioritario atender múltiples problemas de la mayor importancia:

- Realizar una campaña en todos los estados de la República para convencer a las instituciones locales de salud y a las agrupaciones médicas de las bondades y ventajas que ofrecían nuestros procedimientos. Para ello realicé visitas a todas las entidades federativas, previa convocatoria a todos los médicos interesados para asistir a reuniones informativas y propositivas. Algunas de ellas fueron ríspidas y complejas, pero en su mayoría se logró una convicción mayoritaria de los asistentes, convicción que poco a poco creció al conocer los resultados de nuestro trabajo.

- Tuve también que viajar para hablar con los gobernadores de los estados para convencerlos de las ventajas de crear una Comisión Estatal de Arbitraje Médico, logrando la respuesta inmediata de algunos de ellos como los de Tabasco, Estado de México, Veracruz y Quintana Roo, entre otros. 
- Otra tarea difícil y urgente fue dialogar con las organizaciones sindicales de las instituciones de atención a la salud con el fin de convencerlos también de nuestro compromiso de imparcialidad para sus representados.

- No menos importante fue dialogar con los líderes de los sindicatos de trabajadores y por tanto, usuarios de los servicios de salud del IMSS y del ISSSTE para convencerlos de que la CONAMED no solaparía ninguna falta profesional de los médicos que afectara a sus agremiados, sino por el contrario, los apoyaría en todos los casos en que se determinara que les asistía la razón. En todo este esfuerzo fue fundamental la campaña de comunicación social sobre las bondades de la institución, la cual se desarrolló a través de los diferentes medios de comunicación masiva y con numerosas entrevistas a los funcionarios de la CONAMED.

- Se realizaron también numerosas reunionescon las autoridades de las instituciones de servicios médicos y de seguridad social para coordinarnos con sus áreas de atención de quejas, ya que sostenían que éstas eran las responsables, pero se les hizo ver que los pacientes muchas veces los veían como juez y parte y que podríamos lograr mejores resultados trabajando coordinadamente.

- Por ello tuve el mayor cuidado y rigor para fomentar y vigilar la imparcialidad en todos los casos que se admitían en la CONAMED, tarea que consideré prioritaria a lo largo de mi desempeño como comisionado.

- Fue muy importante contar con el apoyo del Dr. José Luis Soberanes cuando fue designado presidente de la CNDH, que previamente fue miembro del Consejo de la CONAMED y con quien pude acordar que la CNDH no aceptaría ninguna queja relacionada con la atención médica que no pasara previamente por la CONAMED.

- Trabajamos de manera permanente para mejorar la calidad de nuestras funciones, por lo que organizamos un gran número de cursos y conferencias de especialistas en los temas de conciliación y arbitraje. A título personal realicé más de un centenar de presentaciones sobre el trabajo de la CONAMED sobre ética médica, derecho sanitario, normas jurídicas, relación médico-paciente, etcétera.

Consideramos prioritario editar la Revista CONAMED para difundir tanto opiniones de expertos sobre temas de nuestra competencia así como para informar sobre nuestras tareas y dar cabida también a artículos de investigación y de divulgación. Asimismo, dada la novedad en el mundo de una institución como la nuestra, establecimos un simposio internacional para realizarse cada año con temas torales relacionados con nuestras funciones y atribuciones.

Desde el inicio me pareció importante diseñar un logotipo que nos identificara y para ello convocamos a un concurso del que resultó vencedor el emblema que hasta el momento caracteriza a la institución. Tuve también la oportunidad de viajar a diversos países tanto para informar sobre el método que aplicamos para resolver los conflictos médico-paciente como para conocer sus soluciones en esta materia.

Haber sido el primer comisionado nacional de arbitraje médico ha sido una de las tareas más estimulantes que se me han encomendado para contribuir a la calidad de la atención de la salud y desde luego, una de las mayores distinciones que se me han conferido durante mi vida profesional. 